\title{
Análise da letalidade decorrente de intoxicação por agrotóxicos em agricultores da região Sul do Brasil ${ }^{1}$
}

\section{Analysis of Mortality Due to Pesticide Poisoning in Farmers from the Southern Region of Brazil}

\author{
Rosane Maria KIRCHNER* \\ Jaqueline SILINSKE** \\ Luciana RICHTER ${ }^{* * *}$ \\ Magda Antunes de CHAVES ${ }^{* * * *}$ \\ Mônica Elisa SCHERER ${ }^{* * * * *}$ \\ Joana Kirchner BENETTI ${ }^{* * * * * *}$ \\ Eniva Miladi Fernandes STUMM ${ }^{* * * * * * *}$
}

\begin{abstract}
RESUMO
Os agrotóxicos são utilizados de forma expressiva para combater as doenças e pragas em propriedades rurais. A forma do uso tem gerado danos ao meio ambiente e à saúde do homem, levando muitas vezes a óbito. Esta pesquisa tem por objetivo analisar as circunstâncias e o índice de letalidade decorrente de intoxicação por agrotóxicos em agricultores da região Sul do Brasil. A partir da base de dados do Sistema Nacional de Informações Tóxico-Farmacológicas (SINITOX) para o período de 1999 a 2009, foram construídas séries históricas para casos, óbitos e circunstância de intoxicação (acidental, ocupacional, induzida, ignorada, outros). Para análise e comparação dos resultados, foram utilizados a estatística descritiva e o índice de letalidade. Observou-se que a letalidade no período estudado variou entre 0,9 (RS) e 8,2 (PR), ocorrendo os dois extremos no ano de 2004. Analisando comparativamente os três Estados, o número de óbitos teve percentual maior no PR. Quando observada a circunstância da intoxicação, destaca-se: no RS, de 1999 a 2004, a forma induzida, isto é, premeditada, ocorreu em torno de 30\% dos casos, diminuindo gradativamente nos anos seguintes (28,10\% a 26,4\%); no PR a forma induzida é preocupante, pois oscilou entre $36,23 \%$ a $52,01 \%$ dos casos, sendo que, nos anos de 2001, 2003, 2004, 2006 e 2007, esta forma ficou em torno de 50\%, explicando o maior índice de letalidade neste Estado; em

\footnotetext{
${ }^{1}$ Projeto PIBIC financiado pelo CNPq.

"Doutora em Engenharia Elétrica (PUC-Rio). Professora da Universidade Federal de Santa Maria (UFSM). E-mail: rosanekirchner@gmail.com

"* Administradora (UFSM). Mestranda em Administração pela Universidade Federal de Santa Maria (UFSM). E-mail: jaquelinesilinske@yahoo.com.br

*** Mestre em Educação (UFSM). Professora da Universidade Federal de Santa Maria (UFSM). E-mail: lurichter@gmail.com

***** Graduanda em Ciências Biológicas (UFSM).E-mail: magda_antunes@hotmail.com

***** Graduanda em Administração (UFSM).E-mail: mony.escherer@hotmail.com

******* Mestra em Engenharia Civil (UNISINOS). Engenheira Civil da 9a Coordenadoria Regional de Obras Públicas (CRO). E-mail: joanakbenetti@yahoo.com.br ${ }^{* * * * * * * *}$ Mestra em Administração (UFRGS). Doutoranda em Enfermagem na Universidade Federal de São Paulo (UNIFESP) e Professora da Universidade Regional do Noroeste do Estado do Rio Grande do Sul (UNIJUÍ).E-mail: eniva@unijui.edu.br
} 
SC, a forma induzida, nos anos de 2005 a 2007, ficou entre 43,5\% e 45\%. Esta análise demonstra que os agrotóxicos utilizados na lavoura, além de levarem à ocorrência de agravos pelo manuseio inadequado, este também em percentual significativo, servem para atentar sobre a vida de indivíduos.

Palavras-chave: agricultores; agrotóxicos; letalidade.

\begin{abstract}
Pesticides are largely used to fight against diseases and pests that attack farms. The way it has been used is causing damage to the environment and to human beings health, often leading them to death. This research aims at analyzing the index and circumstances of mortality caused by pesticide poisoning in farmers from the southern region of Brazil. From the National System of Toxic-Pharmacological Information (SINITOX) data base, considering the period from 1999 to 2007, historical series were built for cases, deaths and intoxication circumstances (accidental, occupational, induced, ignored, and other causes). For the findings analysis and comparisons we used the descriptive statistics and the lethality index. It was possible to observe that the lethality in the considered period ranged from 0,9 (RS) to 8,2(PR), with both extremes occurring in the year 2004. When comparatively analyzing the three States, the number of deaths was greater in the State of Paraná (PR). But, when observing the intoxication circumstances, the State of Rio Grande do Sul (RS) stands out during the years from 1999 to 2004 for the induced form, that is, premeditated, which occurred in about $30 \%$ of the cases, and gradually decreasing in the following years $(28,10 \%$ a $26,4 \%)$; in PR the induced form is worrying since it varied between $36,23 \%$ and $52,01 \%$ of the cases, and in the years 2001, 2003, 2004, 2006 and 2007, this form was in about 50\% of the cases, which may explain the lethality index to be higher in this State; in the State of Santa Catarina (SC), the induced form during the years 2005 to 2007 was about $43,5 \%$ and $45 \%$. This analysis demonstrates that the pesticides used in farming, besides leading to the occurrence of injuries by improper handling, may also, in significant levels, be used to outrage the life of individuals.
\end{abstract}

Keywords: farmers; pesticides; lethality.

\section{Introdução}

A agricultura é essencial para o crescimento econômico, tanto pelo emprego de recursos quanto pela geração de renda para a população (SACHUK e AUGUSTO, 2008). Nesse contexto, a economia capitalista dominante leva a agricultura a contribuir para a manutenção e o aumento de desordens sociais e ambientais (SOUTO et al., 2011).

Nesse sentido, de acordo com a Lei Federal $n^{0} 7$. 802, de 11/07/1989, consideram-se agrotóxicos produtos químicos destinados a controlar as pragas e doenças que causam danos à produção agrícola. São também denominados agrotóxicos as substâncias empregadas como desfolhantes, dessecantes, estimulantes e inibidoras do crescimento das plantas. O meio ambiente e a população têm sofrido as consequências do aumento da utilização de substâncias químicas (HOSHINO et al., 2009). Em contrapartida, o benefício da utilização de agrotóxicos está associado ao crescimento da produção da lavoura, aumentando a produtividade por área colhida (VEIGA, 2007).

Segundo Bedor et al. (2007), existem mais de 2 mil tipos de agrotóxicos disponíveis atualmente no mercado, os quais podem ser classificados, de acordo com os princípios ativos, em organofosforados e organoclorados. Diante da grande variedade de agrotóxicos, Veiga (2007) ressalta que os mesmos são utilizados pelos agricultores para tentar compensar a diminuição de produtividade ocasionada pela degradação do solo e para controlar o surgimento de doenças.

A partir da década de 1960, começou a difundir-se no terceiro mundo o modelo agrícola da "Revolução Verde" e no Brasil puderam ser observadas grandes modificações na agricultura, no ambiente e na saúde humana, 
baseando-se no aumento da utilização de agroquímicos visando aumentar a produtividade agrícola (SOUSA et al., 2011). Isto consistia na adoção de pacotes tecnológicos de fertilizantes e defensivos agrícolas, em variedades melhoradas geneticamente, mecanização e crescimento da fronteira agrícola (MARTINS et al., 2011).

A utilização de agrotóxicos tem o objetivo de maximizar a eficiência econômica e elevar a produtividade rural (VEIGA, 2007). A agricultura alterou sua maneira de produção, alteração ocasionada pela utilização de agroquímicos. Com isso, houve um crescimento significativo de produtividade e a agricultura modernizada se tornou dependente de insumos químicos (SOUSA et al., 2011).

Hoje o Brasil é o país que mais consome agrotóxicos, com um número expressivo de trabalhadores expostos.

No Brasil, o agravamento da contaminação humana e ambiental é gerado pelo grande emprego de agrotóxicos, aliado ao desconhecimento por parte dos usuários em relação aos riscos da utilização desses produtos, à desobediência às normas básicas de segurança, à livre negociação, à ampla pressão por parte das organizações distribuidoras e produtoras e às dificuldades sociais apresentadas no meio rural (MOREIRA et al., 2002). Segundo Bittencourt (2011), o uso indiscriminado de agroquímicos durante anos tem gerado um aglomerado de resíduos nocivos à água, ao solo e ao ar. Confirmando isso, Sousa et al. (2011) afirmam que o solo, as nascentes e rios, poços, açudes, lagos, bem como todos os organismos vivos que dependem destes meios, estão expostos a risco de contaminação. Para os mesmos autores, os efeitos podem alterar a expectativa de vida, fisiologia, crescimento, comportamento e reprodução dos organismos, bem como interferir na disponibilidade de habitats, de alimentos e na biodiversidade, abrangendo os efeitos sobre os inimigos que controlam naturalmente as pragas e provocando a resistência induzida aos próprios agrotóxicos.

Os autores Peres e Moreira (2007) afirmam que os impactos causados pela utilização de agrotóxicos na saúde humana são temas tratados na comunidade científica, principalmente em lugares onde há vasta utilização de agrotóxicos na produção agrícola, sendo os agrotóxicos agentes químicos que resultam em efeitos nocivos à saúde humana (PERES e MOREIRA, 2007).

No Brasil, o ambiente e a saúde do trabalhador rural acabam sofrendo consequências pelo uso de agrotóxicos (MONQUERO et al., 2009) que estão associadas ao uso impróprio, à alta toxicidade dos produtos, à não utilização dos equipamentos de proteção individual e à precariedade dos mecanismos de vigilância (OLIVEIRA-SILVA et al., 2001).

Conforme ILO/WHO (2005), uma das mais perigosas ocupações na atualidade é o trabalho agrícola. Os agrotóxicos geram muitos riscos ocupacionais relacionados a intoxicação aguda, doenças crônicas, problemas reprodutivos, além de danos ambientais, sendo que existem dois tipos de efeitos toxicológicos na saúde humana: por meio direto, quando há intoxicação do agricultor, e por meio indireto, quando o consumidor ingere um alimento cujo nível residual é prejudicial à saúde (SOARES e PORTO, 2007). Segundo Stumm et al. (2010), existem tanto efeitos agudos quanto crônicos relacionados à intoxicação por agrotóxicos, sendo os efeitos agudos aqueles que aparecem primariamente (espasmos musculares, convulsões, náuseas, desmaios, vômitos, diarreias e dificuldades respiratórias), enquanto os crônicos desenvolvem-se tardiamente após semanas, meses ou até mesmo anos.

Alguns dos motivos da intoxicação de agricultores são: uso descontrolado de agrotóxicos, propagandas, receio de perder na produtividade da colheita, falta de utilização de equipamentos de proteção e conhecimentos insuficientes dos riscos (SILVA e FAY, 2004). O nível de instrução dos trabalhadores, o descaso com a segurança ao utilizar os agrotóxicos, o armazenamento, a destinação das embalagens e resíduos desses produtos afetam a eficácia da aplicação, aumentam os riscos de acidentes e justificam em parte os casos de intoxicação aguda e óbitos (POLASTRO, 2005).

Considerando o exposto, este estudo tem por objetivo analisar as circunstâncias e o índice de letalidade decorrente de intoxicação por agrotóxicos em agricultores do Brasil e da região Sul do Brasil, para o período de 1999 a 2009. 


\section{Metodologia}

A pesquisa caracteriza-se como quantitativa descritiva com dados provenientes da base do Sistema Nacional de Informações Tóxico-Farmacológicas (SINITOX). O SINITOX é atualmente composto por 36 Centros de Informação e Assistência Toxicológica (CIT), localizados em 19 estados brasileiros e também no Distrito Federal (FARIA, FASSA e FACHINI, 2007).

No estudo utilizaram-se séries históricas dos casos, óbitos e circunstâncias de intoxicação (acidental, ocupacional, induzida, ignorada, outros) por agrotóxicos em agricultores no período de 1999 a 2009. As mesmas se referem ao ocorrido no Brasil, total da Região Sul e separadamente dos Estados do Rio Grande do Sul, Paraná e Santa Catarina. Para a análise dos dados utilizou-se estatística descritiva, correlação de Pearson e o índice de letalidade. O software estatístico utilizado foi o EXCEL.

\section{Resultados e discussão}

O Brasil é um grande consumidor de agrotóxicos. Entretanto, mesmo que o número de pesquisas brasilei- ras realizadas nos últimos anos a respeito do impacto da utilização de agrotóxicos na saúde humana tenha aumentado, esse número ainda é escasso para avaliar a influência da exposição ocupacional e o tamanho dos danos à saúde advindos do uso intenso de agrotóxicos (NETO et al., 2009).

Conforme Chrisman et al. (2006), um grave problema de saúde pública são os casos de intoxicação humana, sendo importante ressaltar que, no Brasil, é grande a incidência de intoxicações fatais. Corroborando isso, Faria et al. (2004) expõem dados do SINITOX para o ano de 2000, quando os pesticidas de uso agrícola foram responsáveis por 7,0\% das intoxicações e 37,0\% dos óbitos por intoxicações.

No Brasil, segundo Castro e Confalonieri (2005), o difícil acesso dos trabalhadores aos centros de atendimento médico-hospitalar faz com que inúmeras vítimas de acidentes graves acabem morrendo sem assistência médica. Vale ressaltar que o Ministério da Saúde estima que, para cada evento de intoxicação por agrotóxico notificado, há outros 50 não notificados (PERES et al., 2001).

Na Tabela 1 pode ser observado o número de casos, óbitos e índice de letalidade decorrente da intoxicação por agrotóxicos em agricultores no Brasil e nos Estados na Região Sul do Brasil. Na análise do número de casos,

TABELA 1 - NÚMERO DE CASOS, ÓBITOS E ÍNDICE DE LETALIDADE DECORRENTE DA INTOXICAÇÃO POR AGROTÓXICOS EM AGRICULTORES NOS ESTADOS NA REGIÃO SUL DO BRASIL

\begin{tabular}{|l|c|c|c|c|c|c|c|c|c|c|c|c|c|c|c|c|}
\hline & \multicolumn{4}{|c|}{ Brasil** } & \multicolumn{3}{|c|}{ Rio Grande do Sul } & \multicolumn{3}{c|}{ Santa Catarina } & \multicolumn{4}{|c|}{ Paraná } & \multicolumn{3}{|c|}{ Total da Região Sul } \\
\hline Ano & C & O & IL & C & O & IL & C & O & IL & C & O & IL & C & O & IL \\
\hline 1999 & 4674 & 154 & 3,3 & 768 & 11 & 1,4 & 402 & 11 & 2,7 & 338 & 25 & 7,4 & 1508 & 47 & 3,1 \\
\hline 2000 & 5127 & 141 & 2,8 & 775 & 12 & 1,5 & 469 & 11 & 2,2 & 252 & 14 & 5,3 & 1496 & 37 & 2,5 \\
\hline 2001 & 5384 & 157 & 2,9 & 969 & 14 & 1,4 & 605 & 11 & 1,8 & 306 & 20 & 6,5 & 1880 & 45 & 2,4 \\
\hline 2002 & 5717 & 150 & 2,6 & 944 & 13 & 1,4 & 816 & 17 & 2,1 & 287 & 23 & 8,0 & 2047 & 53 & 2,6 \\
\hline 2003 & 6072 & 182 & 3,0 & 896 & 26 & 2,9 & 497 & 14 & 2,9 & 264 & 12 & 4,5 & 1657 & 52 & 3,1 \\
\hline 2004 & 6103 & 164 & 2,7 & 955 & 9 & 0,9 & 487 & 13 & 2,7 & 243 & 20 & 8,2 & 1685 & 42 & 2,5 \\
\hline 2005 & 6249 & 202 & 3,2 & 961 & 17 & 1,8 & 655 & 15 & 2,3 & 276 & 9 & 3,3 & 1892 & 41 & 2,2 \\
\hline 2006 & 6346 & 190 & 3,0 & 876 & 12 & 1,4 & 615 & 9 & 1,5 & 263 & 6 & 2,3 & 1754 & 27 & 1,5 \\
\hline 2007 & 6260 & 209 & 3,3 & 894 & 14 & 1,6 & 557 & 14 & 2,5 & 298 & 19 & 6,4 & 1749 & 47 & 2,7 \\
\hline 2008 & 4243 & 157 & 3,7 & 806 & 19 & 2,4 & $*$ & $*$ & $*$ & 333 & 18 & 5,4 & 1139 & 37 & 3,2 \\
\hline 2009 & 3813 & 115 & 3,0 & 765 & 14 & 1,8 & $*$ & $*$ & $*$ & 293 & 15 & 5,1 & 1058 & 29 & 2,7 \\
\hline
\end{tabular}

$\mathrm{C}=$ Casos; $\mathrm{O}=$ Óbitos; IL = Índice de Letalidade.

${ }^{*}$ Dado numérico não disponível.

*** Dado numérico não computado para o Brasil: Estado do Mato Grosso (não disponível) nos anos de 2007, 2008, 2009; Estado de Santa Catarina (não disponível) nos anos de 2008 e 2009.

Não existe relação significativa $(\mathrm{p}>0,05)$ entre a área plantada em cada Estado da Região Sul, bem como a sua área total e a área do Brasil, com seus respectivos índices de letalidade.

FONTE: Elaborada pelos autores com base no Sistema Nacional de Informações Tóxico-Farmacológicas (SINITOX). 
verifica-se que na série de anos estudada a menor ocorrência foi no último ano (2009) para o Brasil, no RS e também para o total da região Sul.

O trabalhador convencional está exposto a acidentes de trabalho; adicionalmente, os agricultores estão sujeitos a produtos químicos e agrotóxicos. Além disso, é difícil a vigilância de medidas que previnam a segurança do trabalhador, pois o mesmo nem sempre pode ser supervisionado diretamente (SEIFERT e SANTIAGO, 2009). Para haver segurança do trabalho com agrotóxicos é recomendada a utilização de Equipamentos de Proteção Individual (EPIs), os quais controlam exposições dérmicas e respiratórias (OLIVEIRA e MACHADO NETO, 2005). Em estudo realizado na bacia hidrográfica do Rio Dourado, também denominada Vale do Dourado, no Município de Erechim, que pertence à região do Alto Uruguai, Norte do Rio Grande do Sul, percebeu-se que $78 \%$ dos agricultores dizem que utilizam EPIs; no entanto, houve casos de intoxicação, demonstrando que esses equipamentos podem não ser utilizados adequadamente, fato provavelmente associado à falta de treinamento (SOUZA et al., 2010).

Ainda na Tabela 1, observa-se que a letalidade no período estudado variou entre 0,9 (RS) e 8,2 (PR), ocorrendo os dois extremos no ano de 2004. Se observado o conjunto de anos, o índice de letalidade foi maior na maioria dos anos no Estado do PR e menor no RS. Separadamente, para os Estados do RS e de SC o maior índice de letalidade foi no ano de 2003, no PR foi em 2004 e, considerando o Brasil todo, foi no ano de 2008.

Os trabalhadores rurais são um grupo populacional bastante vulnerável aos efeitos danosos provocados pelos agrotóxicos (PERES et al., 2003 apud BEDOR et al., 2009). Muitas vezes, as informações disponíveis sobre os cuidados e precauções com o uso de agrotóxicos são inatingíveis aos trabalhadores rurais, que têm dificuldades de interpretá-las e, assim, acabam adotando práticas de trabalho perigosas (PERES e MOREIRA, 2007). Segundo estes autores, o problema de contaminação por agrotóxicos é grave, isso devido à diversidade de questões que determinam essa situação, sejam elas de ordem social, econômica e/ou cultural.

$\mathrm{Na}$ Figura 1, observa-se visualmente o índice de letalidade dos Estados da Região Sul e do Brasil. Os Estados do Rio Grande do Sul e de Santa Catarina possuem em todos os anos a letalidade inferior comparativamente ao do Brasil; em contrapartida, o Paraná, em todos os anos exceto o de 2006, possui os índices de letalidade superiores aos do Brasil.

Quando realizada a correlação entre a área plantada em cada Estado da região Sul, bem como a área total e a área do Brasil, com seus respectivos índices de letalidade, observou-se que não existe relação significativa entre as variáveis $(p>0,05)$. Logo, a alteração de área de plantio não está relacionada ao índice de letalidade.

No ambiente rural, os EPI mais utilizados são máscaras protetoras, óculos, luvas impermeáveis, macacão com mangas compridas e aventais impermeáveis. Os EPIs devem possuir Certificado de Aprovação do Ministério do Trabalho, ser indicados em receituários agronômicos e nos rótulos dos produtos (BARBOSA e MACHADO, 2010). Porém, a utilização de EPIs modernos e em perfeita condição de funcionamento não é suficiente para o não acontecimento de acidentes com indivíduos expostos a agrotóxicos (POLASTRO, 2005).

Portanto, Stumm et al. (2010) afirmam que a não utilização ou a utilização incompleta das medidas de proteção proporciona perigo à saúde do trabalhador rural, estando relacionada aos altos índices de intoxicações por agrotóxicos.

Os acidentes com agrotóxicos estão relacionados ao efeito dessas substâncias, que possuem o objetivo de diminuir as doenças da lavoura. Desse modo, o ser humano acaba contaminando seu local de trabalho, atingindo os trabalhadores, a produção e o meio ambiente (PIGNATI et al., 2007).

Grande parte dos estudos realizados no Brasil aponta contaminações diferenciadas, tais como humana (ocupacional, acidental ou suicida) e ambiental, não avaliando a grande variabilidade das causas do problema de uma maneira integrada (MOREIRA et al., 2002).

Na Tabela 2, verifica-se o número de ocorrências anuais de intoxicação de agricultores das regiões em estudo segundo a circunstância em que a mesma ocorreu. O maior percentual no Brasil foi a forma "tentativa de suicídio", sendo que esta variou de 35,79\% até 48,29\%. Ainda, no Brasil, as formas "ocupacional" e "acidental" também destacaram-se pelos altos percentuais de casos ocorridos de intoxicação por agrotóxicos em agricultores. Na região Sul, houve predominância das circunstâncias "acidental", "ocupacional" e "tentativa de suicídio". 


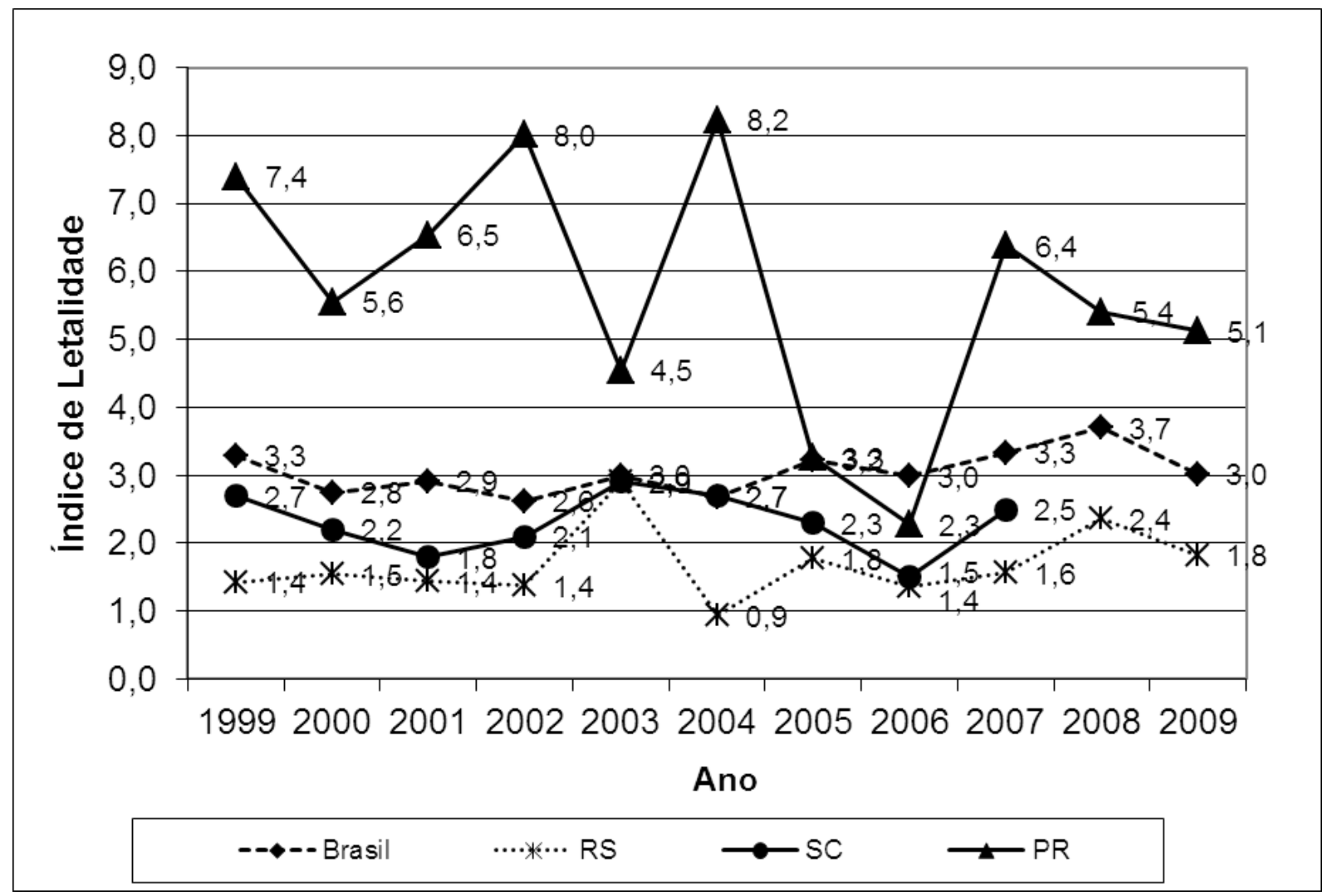

FIGURA 1 - Índice de letalidade decorrente da intoxicação por agrotóxicos em agricultores nos Estados na Região Sul do Brasil FONTE: Elaborada pelos autores com base no Sistema Nacional de Informações Tóxico-Farmacológicas (SINITOX).

Peres e Moreira (2007), em seu estudo, perceberam que os agrotóxicos acarretam agravos à saúde em decorrência do manuseio impróprio e também são empregados para atentar sobre a vida de pessoas com problemas psicológicos. Para os mesmos autores, diversos "distúrbios do sistema nervoso foram associados à exposição aos agrotóxicos organofosforados, principalmente aqueles ligados à neurotoxicidade destes produtos, observados através de efeitos neurológicos retardados" (PERES e MOREIRA, 2007, p. 614). Também Meyer, Resende e Abreu (2007), em uma pesquisa com objetivo de avaliar a incidência e as características de suicídios e de intoxicações por agrotóxicos no município de Luz (MG), bem como a situação da utilização desses agentes por um grupo de moradores da zona rural, mostra que a incidência de suicídio neste município, de 2000 a 2004, foi de 13,2 suicídios por agrotóxicos/100.000 hab./ano, verificando que os sintomas relatados nos prontuários, com maior frequência, foram náuseas, vômitos, erupções cutâneas, perda da memória e depressão.

Em estudo abordado por Pires, Caldas e Recena. (2005), foram realizadas correlações entre a prevalência de intoxicações e tentativas de suicídio, demonstrando a atividade agrícola em culturas temporárias, percentual de pequenas propriedades, predominância de intoxicações e tentativas de suicídio, evidenciando os municípios de Dourados, Fátima do Sul e Vicentina como pontos críticos na microrregião de Dourados, no Estado do Mato Grosso do Sul. 
TABELA 2 - CIRCUNSTÂNCIA DA INTOXICAÇÃO POR AGROTÓXICOS EM AGRICULTORES NO BRASIL E REGIÃO SUL

\begin{tabular}{|c|c|c|c|c|c|c|c|c|}
\hline & Ano & $\begin{array}{c}\text { A } \\
\text { n (\%) }\end{array}$ & $\begin{array}{c}\mathrm{Oc} \\
\mathrm{n}(\%)\end{array}$ & $\begin{array}{c}\text { TS } \\
\text { n }(\%)\end{array}$ & $\begin{array}{c}\mathrm{Vi} \\
\mathrm{n}(\%)\end{array}$ & $\begin{array}{c}\mathrm{Ig} \\
\mathrm{n}(\%)\end{array}$ & $\begin{array}{c}\mathrm{O} \\
\mathrm{n}(\%)\end{array}$ & $\begin{array}{c}\mathrm{T} \\
\mathrm{n}(\%)\end{array}$ \\
\hline \multirow{11}{*}{ Brasil } & 1999 & $1331(28,48)$ & $1499(32,07)$ & $1673(35,79)$ & $10(0,21)$ & $96(2,05)$ & $65(1,39)$ & $4674(100)$ \\
\hline & 2000 & $1663(32,44)$ & $1378(26,88)$ & $1933(37,70)$ & $11(0,21)$ & $88(1,72)$ & $54(1,05)$ & $5127(100)$ \\
\hline & 2001 & $1782(33,10)$ & $1370(25,45)$ & $2019(37,50)$ & $38(0,71)$ & $62(1,15)$ & $113(2,10)$ & $5384(100)$ \\
\hline & 2002 & $1581(27,65)$ & $1792(31,35)$ & $2095(36,65)$ & $36(0,63)$ & $107(1,87)$ & $155(1,85)$ & $5717(100)$ \\
\hline & 2003 & $1778(29,28)$ & $1750(28,82)$ & $2281(37,57)$ & $22(0,36)$ & $139(2,29)$ & $102(1,68)$ & $6072(100)$ \\
\hline & 2004 & $1550(25,40)$ & $1763(28,89)$ & $2504(41,03)$ & $30(0,49)$ & $165(2,70)$ & $91(1,49)$ & $6103(100)$ \\
\hline & 2005 & $1454(23,27)$ & $1790(28,64)$ & $2696(43,14)$ & $31(0,50)$ & $142(2,27)$ & $136(2,18)$ & $6249(100)$ \\
\hline & 2006 & $1428(22,50)$ & $1927(30,37)$ & $2710(42,70)$ & $53(0,84)$ & $96(1,51)$ & $132(2,08)$ & $6346(100)$ \\
\hline & 2007 & $1472(23,51)$ & $1564(24,98)$ & $2899(46,31)$ & $43(0,69)$ & $138(2,20)$ & $144(2,30)$ & $6260(100)$ \\
\hline & 2008 & $1000(23,57)$ & $986(23,24)$ & $2049(48,29)$ & $40(0,94)$ & $107(2,52)$ & $61(1,44)$ & $4243(100)$ \\
\hline & 2009 & $971(25,47)$ & $926(24,29)$ & $1719(45,08)$ & $24(0,63)$ & $105(2,75)$ & $68(1,78)$ & $3813(100)$ \\
\hline \multirow{11}{*}{$\begin{array}{c}\text { Região } \\
\text { Sul }\end{array}$} & 1999 & $598(39,66)$ & $375(24,87)$ & $483(32,03)$ & $4(0,27)$ & $27(1,79)$ & $21(1,39)$ & $1508(100)$ \\
\hline & 2000 & $639(42,71)$ & $321(21,46)$ & $505(33,76)$ & $1(0,00)$ & $24(1,60)$ & $7(0,47)$ & $1496(100)$ \\
\hline & 2001 & $660(35,11)$ & $480(25,53)$ & $637(33,88)$ & $23(1,22)$ & $33(1,76)$ & $47(2,50)$ & $1880(100)$ \\
\hline & 2002 & $806(39,37)$ & $459(22,42)$ & $693(33,85)$ & $9(0,44)$ & $25(1,22)$ & $55(2,69)$ & $2047(100)$ \\
\hline & 2003 & $573(34,58)$ & $426(25,71)$ & $587(35,43)$ & $7(0,42)$ & $5(0,30)$ & $59(3,56)$ & $1657(100)$ \\
\hline & 2004 & $573(34,01)$ & $484(28,72)$ & $563(33,41)$ & $4(0,24)$ & $41(2.43)$ & $20(1,19)$ & $1685(100)$ \\
\hline & 2005 & $536(28,33)$ & $595(31,45)$ & $651(34,41)$ & $4(0,21)$ & $60(3,17)$ & $46(2,43)$ & $1892(100)$ \\
\hline & 2006 & $429(24,46)$ & $620(35,35)$ & $649(37,00)$ & $6(0,34)$ & $25(1,43)$ & $25(1,43)$ & $1754(100)$ \\
\hline & 2007 & $417(23,84)$ & $625(35,74)$ & $626(35,79)$ & $7(0,40)$ & $45(2,57)$ & $29(1,66)$ & $1749(100)$ \\
\hline & 2008 & $287(25,20)$ & $379(33,27)$ & $407(35,73)$ & $19(1,67)$ & $37(3,25)$ & $10(0,88)$ & $1139(100)$ \\
\hline & 2009 & $288(27,22)$ & $369(34,88)$ & $378(35,73)$ & $3(0,28)$ & $7(0,66)$ & $13(1,23)$ & $1058(100)$ \\
\hline
\end{tabular}

A = Acidental Individual, Acidental Coletivo, Acidental Ambiental; Oc = Ocupacional; TS = Tentativa de Suicídio; Vi = Violência/Suicídio Ig = Ignorados; O = Outros, Uso Terapêutico, Presc. Méd. Inadequada, Erro de Administração, Automedicação, Abstinência, Abuso, Ingestão de Alimentos, Tentativa de Aborto; T = Total.

FONTE: Elaborada pelos autores com base no Sistema Nacional de Informações Tóxico-Farmacológicas(SINITOX).

No período de estudo na microrregião de Dourados notou-se que ocorreram 475 intoxicações por uso de agrotóxicos, sendo que 261 foram acidentais ou profissionais, 203 foram tentativas de suicídio e 11 indeterminadas. Logo, 14 pessoas foram a óbito causado pela intoxicação e 63 pela ingestão voluntária (PIRES, CALDAS e RECENA, 2005).

Quando estudada a circunstância da intoxicação, destaca-se: no RS, de 1999 a 2003, a forma Acidental, variando entre $47,92 \%$ a $33,93 \%$; entre 2004 a 2009 , destacou-se a forma Ocupacional, variando de $36,23 \%$ a 44,18\%; no PR, a forma Tentativa de Suicídio é preocupante, pois oscilou entre $36,23 \%$ e $51,54 \%$ dos casos, sendo que nos anos de 2001, 2003, 2004, 2006, 2007,
2008 e 2009 esta forma ficou em torno de $50 \%$; em SC, a forma Acidental nos anos de 1999 a 2004 ficou entre $40,55 \%$ e $45,17 \%$ e, no período de 2005 a 2007, prevaleceu a Tentativa de Suicídio, que ficou entre 42,9\% e $44,23 \%$ (Tabela 3 ).

A utilização dos agrotóxicos tem trazido uma série de consequências, tanto para o ambiente como para a saúde do trabalhador rural, as quais são relacionadas ao uso inadequado dessas substâncias, à pressão exercida pela indústria e pelo comércio para esta utilização, à alta toxicidade de certos produtos, à ausência de informações sobre saúde e segurança de fácil apropriação por parte deste grupo de trabalhadores e à precariedade dos mecanismos de vigilância (PERES et al., 2005). 
KIRCHNER, R. M. et al. Análise da letalidade decorrente de intoxicação por agrotóxicos em agricultores da região Sul do Brasil

TABELA3 - CIRCUNSTÂNCIA DA INTOXICAÇÃO POR AGROTÓXICOS EM AGRICULTORES NO ESTADO DO RIO GRANDE DO SUL

\begin{tabular}{|c|c|c|c|c|c|c|c|c|}
\hline \multirow[b]{2}{*}{ Estado } & \multirow[b]{2}{*}{ Ano } & \multicolumn{7}{|c|}{ Circunstância } \\
\hline & & $\begin{array}{c}\text { A } \\
\mathrm{n}(\%)\end{array}$ & $\begin{array}{c}\mathrm{Oc} \\
\mathrm{n}(\%)\end{array}$ & $\begin{array}{c}\mathrm{TS} \\
\mathrm{n}(\%)\end{array}$ & $\begin{array}{c}\mathrm{Vi} \\
\mathrm{n}(\%)\end{array}$ & $\begin{array}{c}\mathrm{Ig} \\
\mathrm{n}(\%)\end{array}$ & $\begin{array}{c}\mathrm{O} \\
\mathrm{n}(\%)\end{array}$ & $\begin{array}{c}\mathrm{T} \\
\mathrm{n}(\%)\end{array}$ \\
\hline & 1999 & $368(47,92)$ & $147(19,14)$ & $231(30,08)$ & $1(0,13)$ & $12(1,56)$ & $9(1,17)$ & $768(100)$ \\
\hline & 2000 & $369(47,61)$ & $152(19,61)$ & $233(30,06)$ & $0(0,00)$ & $17(2,19)$ & $4(0,52)$ & $775(100)$ \\
\hline & 2001 & $337(34,78)$ & $290(29,93)$ & $280(28,90)$ & $12(1,24)$ & $21(2,17)$ & $29(2,99)$ & $969(100)$ \\
\hline & 2002 & $377(39,94)$ & $231(24,47)$ & $307(32,52)$ & $0(0,00)$ & $11(1,16)$ & $18(1,91)$ & $944(100)$ \\
\hline \multirow[t]{11}{*}{$\mathrm{RS}$} & 2003 & $304(33,93)$ & $250(27,90)$ & $302(33,71)$ & $2(0,22)$ & $2(0,22)$ & $36(4,02)$ & $896(100)$ \\
\hline & 2004 & $308(32,25)$ & $346(36,23)$ & $282(29,53)$ & $3(0,31)$ & $6(0,63)$ & $10(1,05)$ & $955(100)$ \\
\hline & 2005 & $264(27,47)$ & $373(38,81)$ & $270(28,10)$ & $0(0,00)$ & $39(4,06)$ & $15(1,56)$ & $961(100)$ \\
\hline & 2006 & $232(26,48)$ & $380(43,38)$ & $251(28,65)$ & $0(0,00)$ & $8(0,91)$ & $5(0,57)$ & $876(100)$ \\
\hline & 2007 & $230(25,73)$ & $395(44,18)$ & $234(26,17)$ & $2(0,22)$ & $23(2,57)$ & $10(1,12)$ & $894(100)$ \\
\hline & 2008 & $219(27,17)$ & $318(39,45)$ & $237(29,40)$ & $2(0,25)$ & $27(3,35)$ & $3(0,37)$ & $806(100)$ \\
\hline & 2009 & $215(28,10)$ & $305(39,87)$ & $227(29,67)$ & $1(0,13)$ & $7(0,92)$ & $10(1,31)$ & $765(100)$ \\
\hline & 1999 & $67(19,82)$ & $122(36,09)$ & $133(39,35)$ & $0(0,00)$ & $11(3,25)$ & $5(1,48)$ & $338(100)$ \\
\hline & 2000 & $58(23,02)$ & $91(36,11)$ & $100(39,68)$ & $0(0,00)$ & $3(1,19)$ & $0(0,00)$ & $252(100)$ \\
\hline & 2001 & $74(24,18)$ & $82(26,80)$ & $147(48,04)$ & $0(0,00)$ & $3(0,98)$ & $0(0,00)$ & $306(100)$ \\
\hline & 2002 & $76(26,48)$ & $78(27,18)$ & $109(37,98)$ & $3(1,05)$ & $5(1,74)$ & $19(5,57)$ & $287(100)$ \\
\hline \multirow[t]{12}{*}{ PR } & 2003 & $56(21,21)$ & $76(28,79)$ & $124(46,97)$ & $4(1,52)$ & $0(0,00)$ & $4(1,52)$ & $264(100)$ \\
\hline & 2004 & $45(18,52)$ & $57(23,46)$ & $118(48,56)$ & $1(0,41)$ & $21(8,64)$ & $1(0,41)$ & $243(100)$ \\
\hline & 2005 & $68(24,64)$ & $96(34,78)$ & $100(36,23)$ & $0(0,00)$ & $5(1,81)$ & $7(2,54)$ & $276(100)$ \\
\hline & 2006 & $37(14,07)$ & $83(31,56)$ & $126(47,91)$ & $1(0,38)$ & $5(1,90)$ & $11(4,18)$ & $263(100)$ \\
\hline & 2007 & $63(21,14)$ & $70(23,49)$ & $152(51,01)$ & $2(0,67)$ & $3(1,01)$ & $8(2,68)$ & $298(100)$ \\
\hline & 2008 & $68(20,42)$ & $61(18,32)$ & $170(51,05)$ & $17(5,11)$ & $10(3,00)$ & $7(2,10)$ & $333(100)$ \\
\hline & 2009 & $73(24,91)$ & $64(21,84)$ & $151(51,54)$ & $2(0,68)$ & $0(0,00)$ & $3(1,02)$ & $293(100)$ \\
\hline & 1999 & $163(40,55)$ & $106(26,37)$ & $119(29,60)$ & $3(0,75)$ & $4(1,00)$ & $7(1,74)$ & $402(100)$ \\
\hline & 2000 & $212(45,20)$ & $78(16,63)$ & $172(36,67)$ & $0(0,00)$ & $4(0,85)$ & $3(0,64)$ & $469(100)$ \\
\hline & 2001 & $249(41,16)$ & $108(17,85)$ & $210(34,71)$ & $11(1,82)$ & $9(1,49)$ & $18(2,98)$ & $605(100)$ \\
\hline & 2002 & $353(43,26)$ & $150(18,38)$ & $277(33,95)$ & $6(0,74)$ & $9(1,10)$ & $20(2,57)$ & $816(100)$ \\
\hline & 2003 & $213(42,86)$ & $100(20,12)$ & $161(32,39)$ & $1(0,20)$ & $3(0,60)$ & $19(3,82)$ & $497(100)$ \\
\hline \multirow[t]{6}{*}{$\mathrm{SC}$} & 2004 & $220(45,17)$ & $81(16,63)$ & $163(33,47)$ & $0(0,00)$ & $14(2,87)$ & $9(1,85)$ & $487(100)$ \\
\hline & 2005 & $204(31,15)$ & $126(19,24)$ & $281(42,90)$ & $4(0,61)$ & $16(2,44)$ & $24(3,66)$ & $655(100)$ \\
\hline & 2006 & $160(26,02)$ & $157(25,53)$ & $272(44,23)$ & $5(0,81)$ & $12(1,95)$ & $9(1,46)$ & $615(100)$ \\
\hline & 2007 & $124(22,26)$ & $160(28,73)$ & $240(43,09)$ & $3(0,54)$ & $19(3,41)$ & $11(1,97)$ & $557(100)$ \\
\hline & 2008 & $*$ & $*$ & $*$ & $*$ & $*$ & $*$ & $*$ \\
\hline & 2009 & $*$ & $*$ & $*$ & $*$ & $*$ & $*$ & $*$ \\
\hline
\end{tabular}

A = Acidental Individual, Acidental Coletivo, Acidental Ambiental; Oc = Ocupacional; TS = Tentativa de Suicídio; Vi = Violência/Suicídio; $\mathrm{Ig}=$ Ignorados; $\mathrm{O}$ = Outros, Uso Terapêutico, Presc. Méd. Inadequada, Erro de Administração, Automedicação, Abstinência, Abuso, Ingestão de Alimentos, Tentativa de Aborto; $\mathrm{T}=$ Total

* Dado numérico não disponível

FONTE: Elaborada pelos autores com base no Sistema Nacional de Informações Tóxico-Farmacológicas (SINITOX) 
De acordo com Konradsen et al. (2003), existe uma busca incessante por tecnologias menos agressivas e perigosas para controlar pragas e doenças que atingem culturas com importância econômica. O controle dos problemas advindos da utilização indiscriminada de agrotóxicos apenas poderá ser obtido com a aceitação de métodos alternativos ou pelo uso seguro e cuidadoso desses produtos (PERES e MOREIRA, 2007). É fundamental reduzir o uso de pesticidas com o intuito de diminuir os casos de acidentes e tentativas de suicídio, podendo gerar redução do risco ocupacional e ambiental (KONRADSEN et al., 2003).

\section{Conclusão}

A partir deste estudo, verificou-se que o maior índice de letalidade em todos os anos ocorreu no Estado do Paraná em comparação aos demais Estados da região Sul do Brasil. Levando-se em consideração as circuns-

\section{Referências}

BARBOSA, L. D. S.; MACHADO, J. G. C. F. Análise dos programas de fomento ao uso de equipamentos de proteção individual das indústrias de defensivos agrícolas. In: ENCONTRO DA SOCIEDADE BRASILEIRA DE ECONOMIA, ADMINISTRAÇÃO E SOCIOLOGIA RURAL, 48., jul. 2010. Campo Grande. Anais.

BEDOR, C. N. G. et al. Avaliação e reflexão da comercialização e utilização de agrotóxicos na região do submédio do vale do São Francisco. Revista Baiana de Saúde Pública, v. 31, n. 1, p. 68-76, 2007.

et al. Vulnerabilidades e situações de risco relacionadas ao uso de agrotóxicos na fruticultura irrigada. Revista Brasileira de Epidemiologia, v. 12, n. 1, p. 39-49, 2009.

BITTENCOURT, E. Embrapa comprova prejuízos aos recursos hídricos por defensivos e pesquisa opções de menor impacto no meio ambiente. 2011. Disponível em: <http:/www.agrisustentavel.com/toxicos/residuorh.htm>. Acesso em: maio 2011.

BRASIL. Lei $n^{\circ}$ 7.802, de 11 de julho de 1989. Disponível em: <http://www.planalto.gov.br/ccivil_03/leis/17802.htm>. Acesso em: jun. 2013. tâncias de intoxicação na região Sul do Brasil, houve predominância na tentativa de suicídio de agricultores por meio de intoxicação. Cabe ressaltar, também, a representatividade dos casos de acidentes individuais, coletivos, ambientais e ocupacionais.

É importante destacar que no Estado do Paraná a circunstância tentativa de suicídio foi a mais alta em comparação a Santa Catarina e Rio Grande do Sul, variando de $36,23 \%$ a $44,18 \%$, resultado considerado preocupante.

As formas acidental individual, coletiva, ambiental e ocupacional também inspiram cuidados, pois na maioria das vezes os agricultores não se protegem adequadamente por meio do emprego de EPIs ou desconhecem o modo correto de utilizá-los.

Esta análise demonstra que os agrotóxicos utilizados na lavoura, além de levarem à ocorrência de agravos à saúde pelo manuseio inadequado, também, em percentual significativo, são utilizados como meio para tentativa de suicídio.

CASTRO, J. S. M.; CONFALONIERI, U. Uso de agrotóxicos no município de Cachoeiras de Macacu (RJ). Ciência e Saúde Coletiva, v. 10, n. 2, p. 473-482, 2005.

CHRISMAN, J. R. et al. Desenvolvimento e análise de um sistema de informações forenses como ferramenta na vigilância das intoxicações por substâncias químicas no âmbito da saúde pública. In: BIENAL DA PESQUISA DA FIOCRUZ, 5., dez. 2006. Rio de Janeiro. Anais.

ESTADO DE SÃO PAULO. Brasil lidera uso mundial de agrotóxicos. 2009. Disponível em: <http://www.estadao.com. br/estadaodehoje/20090807/not_imp414820,0.php.>. Acesso em: nov. 2012.

FARIA, N. M. X.; FASSA, A. G.; FACCHINI, L. A. Intoxicação por agrotóxicos no Brasil: os sistemas oficiais de informação e desafios para realização de estudos epidemiológicos. Ciência e Saúde Coletiva, v. 12, n. 1, p. 25-38, 2007.

et al. Trabalho rural e intoxicações por agrotóxicos. Cadernos de Saúde Pública, v. 20, n. 5, p. 1298-1308, 2004.

ILO/WHO. Joint Press Release ILO/WHO: Number of Work related Accidents and Illnesses Continues to Increase - ILO and 
WHO Join in Call for Prevention Strategies. 2012. Disponível em: $<$ http://www.ilo.org/public/english/bureau/inf/pr/2005/21. htm>. Acesso em: dez. 2012.

HOSHINO, A. C. H. et al. A autopercepção da saúde auditiva e vestibular de trabalhadores expostos a organofosforados. Revista CEFAC, v. 11, n. 4, p. 681-687, 2009.

KONRADSEN, F. et al. Reducing acute poisoning in developing countries: options for restricting the availability of pesticides. Toxicology, v. 192, n .2-3, p. 249-261, 2003.

MARTINS, R.; OLIVETTE, M. P. A.; NACHILUK, K. Sustentabilidade: novos desafios e oportunidades para a produção paulista de cana-de-açúcar. Informações Econômicas, v. 41, n. 2, p. 23-36, 2011.

MEYER, T. N.; RESENDE, I. L. C.; ABREU, J. C. A. Incidência de suicídios e uso de agrotóxicos por trabalhadores rurais em Luz (MG), Brasil. Revista Brasileira de Saúde Ocupacional [periódico na internet]. 2007. Disponível em: $<$ http://www. fundacentro.gov.br/rbso/BancoAnexos/RBSO $\% 20116 \% 20$ Suic\%C3\%ADdios\%20e\%20agrot\%C3\%B3xicos.pdf>. Acesso em: jan. 2012.

MONQUERO, P. A.; INÁCIO, E. M.; SILVA, A. C. Levantamento de agrotóxicos e utilização de equipamento de proteção individual entre os agricultores da região de araras. Arquivos do Instituto Biológico, v. 76, n. 1, p. 135-139, 2009.

MOREIRA J. C. et al. Avaliação integrada do impacto do uso de agrotóxicos sobre a saúde humana em uma comunidade agrícola de Nova Friburgo, RJ. Ciência e Saúde Coletiva, v. 7, n. 2, p. 299-311, 2002.

NETO, A. J. L. et al. Uso de agrotóxicos e utilização de equipamento de proteção individual por produtores no sertão paraibano. Revista Verde, v. 4, n. 4, p. 107-114, 2009.

OLIVEIRA, M. L.; MACHADO NETO, J. G. Segurança na aplicação de agrotóxicos em cultura de batata em regiões montanhosas. Revista Brasileira de Saúde Ocupacional, v. 30, n. 112, p. 15-25, 2005.

OLIVEIRA-SILVA, J. J. et al. Influência de fatores socioeconômicos na contaminação por agrotóxicos, Brasil. Revista de Saúde Pública, v. 35, n. 2, p. 130-135, 2001.

PERES, F.; MOREIRA, J. C. Saúde e ambiente em sua relação com o consumo de agrotóxicos em um polo agrícola do Estado do Rio de Janeiro, Brasil. Cadernos de Saúde Pública, v. 23, n. 4, p. 612-621, 2007.

; CLAUDIO, L. Os impactos dos agrotóxicos sobre a saúde e o ambiente. Ciência e Saúde Coletiva, v. 12, n. 1, p. 4-5, 2007. et al. Comunicação relacionada ao uso de agrotóxicos em região agrícola do Estado do Rio de Janeiro. Revista de Saúde Pública, v. 35, n. 6, p. 564-570, 2001.

et al. Desafios ao estudo da contaminação humana e ambiental por agrotóxicos. Ciência e Saúde Coletiva, v. 10, supl., p. 27-37, 2005.

PIGNATI, W. A. et al. Acidente rural ampliado: o caso das "chuvas" de agrotóxicos sobre a cidade de Lucas do Rio Verde - MT. Ciência e Saúde Coletiva, v. 12, n. 1, p. 105-114, 2007.

PIRES, D. X.; CALDAS, E. D.; RECENA, M. C. P. Intoxicações provocadas por agrotóxicos de uso agrícola na microrregião de Dourados, Mato Grosso do Sul, Brasil, no período de 1992 a 2002. Caderno de Saúde Pública, v. 21, n. 3, p. 804-814, 2005.

POLASTRO, D. Estudo dos casos de intoxicação ocasionados pelo uso de agrotóxicos no Estado do Paraná, durante o período de 1993 a 2000. Piracicaba, Dissertação (Mestrado em Ecologia de Agroecossistemas) - USP, 2005.

SACHUK M. I.; AUGUSTO, C. A. Competência e competitividade na agricultura orgânica em pequenos empreendimentos rurais na região noroeste do Paraná. Revista da Micro e Pequena Empresa, v. 2, n. 2, p. 4-20, 2008.

SEIFERT, A. L.; SANTIAGO, D. C. Formação dos profissionais das áreas de ciências agrárias em segurança do trabalho rural. Ciência e Agrotecnologia, v. 33, n. 4, p. 1131-1138, 2009.

SILVA, L. S. et al. Uso de agrotóxicos em Pernambuco na região de Salgueiro e Petrolina. In: JORNADA DE ENSINO, PESQUISA E EXTENSÃO, JEPEX, UFRPE, 10., 2010. Recife. Anais.

SILVA, M. M. S.; FAY, E. F. Agrotóxicos e ambiente. Brasília: Embrapa Informação Tecnológica, 2004. 480p.

SOARES, W. L.; PORTO, M. F. Atividade agrícola e externalidade ambiental: uma análise a partir do uso de agrotóxicos no cerrado brasileiro. Ciência e Saúde Coletiva, v. 12, n. 1, p. 131-143, 2007.

SOUTO, R. A. et al. Análise da viabilidade ambiental de práticas agroecológicas adotadas por agricultores familiares do município de Lagoa Seca, Paraíba. Engenharia Ambiental, v. 8, n. 1, p. 177-193, 2011.

SOUSA, I.; CHAVES, L. H. G.; JUNIOR, G. B. Uso de agrotóxicos impactando a saúde de horticultores familiares na região de Lagoa Seca, Paraíba. Engenharia Ambiental, v. 8, n. 1, p. 232-245, 2011. 
SOUZA, M. F et al. Riscos de contaminação do ambiente e intoxicação humana pelo uso de agrotóxicos e disposição de resíduos na área rural de Erechim-RS. In: CIC, 19., ENPOS, 12., II Mostra Científica, 2010. Anais.

STUMM E. M. F. et al. Análise do uso de equipamentos de proteção individual por agricultores que utilizam agrotóxicos.
Revista Técnico-Científica de Enfermagem, v. 25, n. 8, p. 203-207, 2010.

VEIGA, M. M. Agrotóxicos: eficiência econômica e injustiça socioambiental. Ciência e Saúde Coletiva, v. 12, n. 1, p. 145$152,2007$.

Recebido em 21 de fevereiro de 2013. Aceito em 05 de agosto de 2013. Publicado em dezembro de 2013. 